ARTICLE

DOI: $10.1038 / s 41467-017-01089-z$

\title{
Two-dimensional metallic tantalum disulfide as a hydrogen evolution catalyst
}

Jianping Shi ${ }^{1}{ }^{2}$, Xina Wang (i) $^{3}$, Shuai Zhang ${ }^{4}$, Lingfeng Xiao ${ }^{3}$, Yahuan Huan ${ }^{1,2}$, Yue Gong ${ }^{5}$, Zhepeng Zhang ${ }^{2}$, Yuanchang $\mathrm{Li}^{4}$, Xiebo Zhou ${ }^{1,2}$, Min Hong',2, Qiyi Fang1,2, Qing Zhang D 1, Xinfeng Liu ${ }^{4}$, Lin Gu 5,6,7, Zhongfan Liu $^{1} \&$ Yanfeng Zhang ${ }^{1,2}$

Two-dimensional metallic transition metal dichalcogenides are emerging as prototypes for uncovering fundamental physical phenomena, such as superconductivity and charge-density waves, as well as for engineering-related applications. However, the batch production of such envisioned transition metal dichalcogenides remains challenging, which has hindered the aforementioned explorations. Herein, we fabricate thickness-tunable tantalum disulfide flakes and centimetre-sized ultrathin films on an electrode material of gold foil via a facile chemical vapour deposition route. Through temperature-dependent Raman characterization, we observe the transition from nearly commensurate to commensurate charge-density wave phases with our ultrathin tantalum disulfide flakes. We have obtained high hydrogen evolution reaction efficiency with the as-grown tantalum disulfide flakes directly synthesized on gold foils comparable to traditional platinum catalysts. This work could promote further efforts for exploring new efficient catalysts in the large materials family of metallic transition metal dichalcogenides, as well as exploiting their applications towards more versatile applications.

\footnotetext{
${ }^{1}$ Department of Materials Science and Engineering, College of Engineering, Peking University, Beijing 100871, China. ${ }^{2}$ Center for Nanochemistry (CNC), Beijing Science and Engineering Center for Nanocarbons, Beijing National Laboratory for Molecular Sciences, College of Chemistry and Molecular Engineering, Peking University, Beijing 100871, China. ${ }^{3}$ Hubei Collaborative Innovation Center for Advanced Organic Chemical Materials, Faculty of Physics and Electronic Technology, Hubei University, Wuhan 430062, China. ${ }^{4}$ Division of Nanophotonics, CAS Key Laboratory of Standardization and Measurement for Nanotechnology, CAS Center for Excellence in Nanoscience, National Center for Nanoscience and Technology, Beijing 100190, China. ${ }^{5}$ Beijing National Laboratory for Condensed Matter Physics, Institute of Physics, Chinese Academy of Sciences, Beijing 100190, China. ${ }^{6}$ Collaborative Innovation Center of Quantum Matter, Beijing 100190, China. ${ }^{7}$ School of Physical Sciences, University of Chinese Academy of Sciences, Beijing 100190, China. Correspondence and requests for materials should be addressed to Y.Z. (email: yanfengzhang@pku.edu.cn)
} 
T wo-dimensional (2D) metallic transition metal dichalcogenides (MTMDCs) such as $\mathrm{TiSe}_{2}{ }^{1-5}, \mathrm{NbSe}_{2}{ }^{6-9}, \mathrm{TaS}_{2}{ }^{10-13}$, and $\mathrm{TaSe}_{2}{ }^{14-16}$, have kindled worldwide research interest due to their rich phase diagrams that include superconductivity, charge-density wave (CDW) and metal-insulator transitions. These intriguing properties are mainly attributed to their reduced dimensionality and the induced quantum confinement effect. Recently, such unique 2D systems have become appealing platforms for exploring the origin of superconductivity and CDW, longstanding puzzles in condensed matter physics ${ }^{17-19}$. For instance, the coexistence of CDW order and superconductivity has been unveiled in atomically thin $\mathrm{TaS}_{2}{ }^{11,12,20}$. However, the $\mathrm{TaS}_{2}$ samples reported in a majority of the publications were obtained by an exfoliation method ${ }^{10-13,20}$, which is time-consuming, incompatible with batch production, and affords little control over thickness and domain size.

Chemical vapour deposition (CVD), compatible with common tool sets and scalable syntheses, has been regarded as a swift and effective route for growing semiconducting TMDCs (e.g. $\mathrm{MoS}_{2}{ }^{21-23}, \mathrm{MoSe}_{2}{ }^{24,}{ }^{25}, \mathrm{WS}_{2}{ }^{26}, 27, \mathrm{ReS}_{2}{ }^{28,}$, 29 , etc.). Very recently, this approach has been extended to the synthesis of MTMDCs $s^{30-32}$. For example, Lou et al. synthesized metallic $\mathrm{VS}_{2}$ single-crystal flakes on $\mathrm{SiO}_{2} / \mathrm{Si}$ through an atmospheric pressure
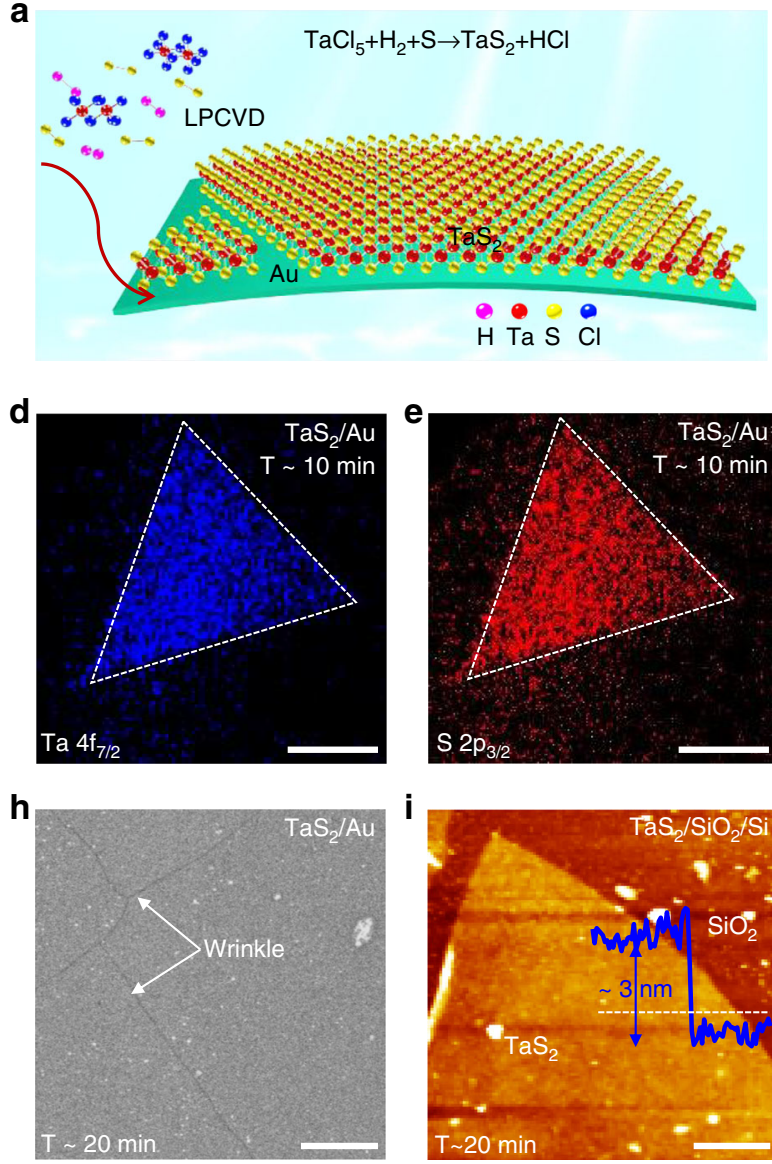

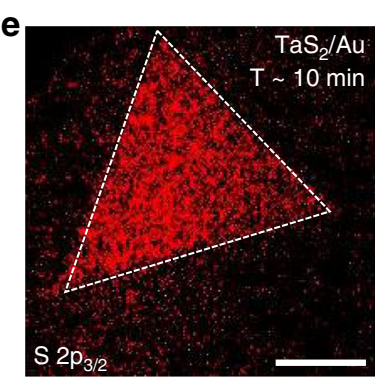

i

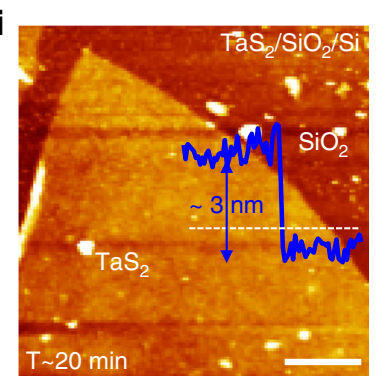

CVD (APCVD) route, with thicknesses ranging from $~ 100$ to $\sim 1100 \mathrm{~nm}^{30}$. Subsequently, Zhang et al. optimized the growth conditions and obtained $\sim 8$-nm-thick $\mathrm{VS}_{2}$ flakes ${ }^{31}$. Meanwhile, Liu et al. reported the APCVD growth of $1 \mathrm{~T}-\mathrm{TaS}_{2}$ flakes on $\mathrm{SiO}_{2} /$ $\mathrm{Si}$ with a wide thickness range of $2 \sim 220 \mathrm{~nm}^{32}$. Nevertheless, large-area syntheses of full coverage or large-domain MTMDCs and identification of their possible applications are still works-inprogress.

Experimental and theoretical efforts have indicated that $\mathrm{MoS}_{2}$ nanoparticles or nanosheets are potential electrocatalysts for the hydrogen evolution reaction (HER) ${ }^{33-35}$, and metallic $1 \mathrm{~T}-\mathrm{MoS}_{2}$ can be much more active than its semiconducting counterpart ${ }^{36}$. However, such 1T-MoS 2 is vulnerable to ambient conditions, and its direct synthesis through reactive alkyl lithium intercalation is difficult. It thus appears reasonable to seek phase-stable MTMDCs to replace $1 \mathrm{~T}-\mathrm{MoS}_{2}$ for realizing efficient catalytic applications. Recent theoretical calculations have predicted the possibility of $\mathrm{TaS}_{2}$ as an active and stable electrocatalyst ${ }^{37}$. From the experimental side, Chen et al. performed HER measurements of liquid-phase-exfoliated $1 \mathrm{~T}-\mathrm{TaS}_{2}$, and reported an enhanced catalytic activity for atomic-scale-pore decorated $\mathrm{TaS}_{2}$ (introduced via oxygen plasma treatment) over the conventional form $^{38}$. However, the reported HER performance was still not
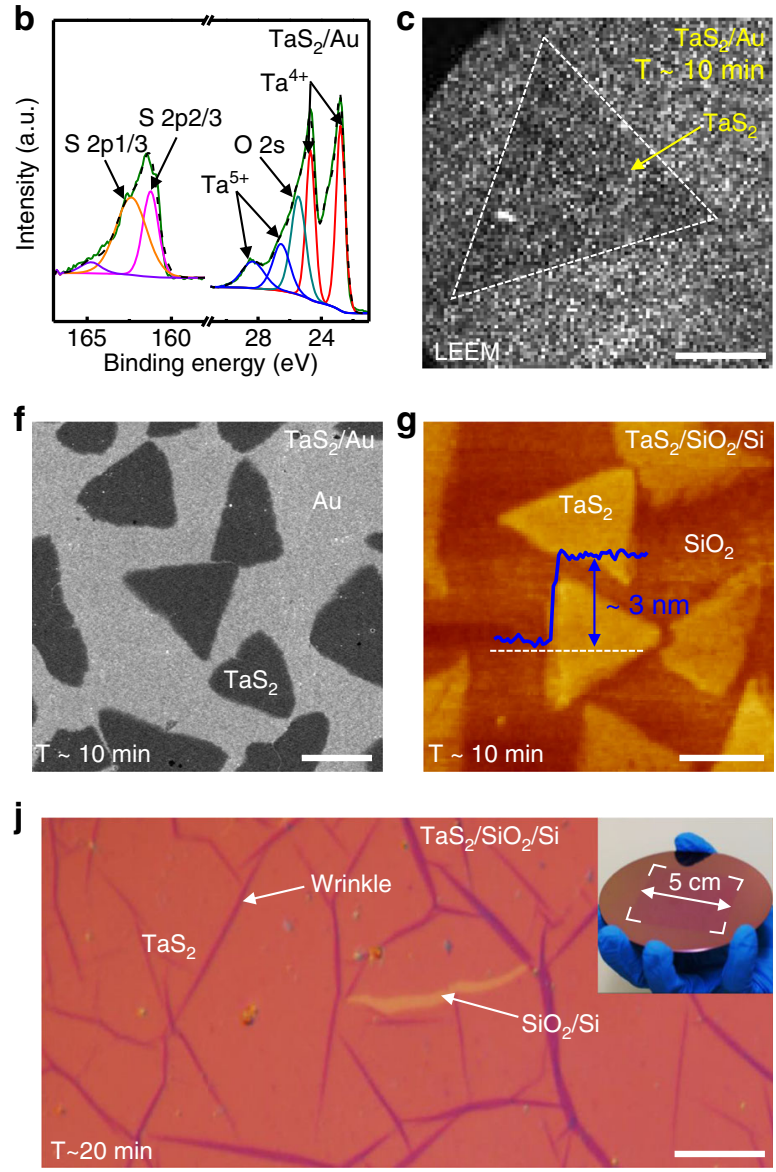

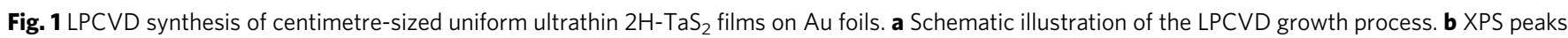
of $\mathrm{Ta}$ and $\mathrm{S}$ in as-grown $2 \mathrm{H}-\mathrm{TaS}_{2}$, respectively. c-e Synchrotron radiation-based $\mathrm{LEEM}$ and $\mu$-XPS elemental mapping of Ta $\left(4 \mathrm{f}_{7 / 2}\right)$ and $\mathrm{S}\left(2 \mathrm{p}_{3 / 2}\right)$ acquired on consecutive areas of $20 \times 20 \mu \mathrm{m}^{2}$ (synthesized at $\sim 750^{\circ} \mathrm{C}$ for $\sim 10$ min under $\mathrm{Ar} / \mathrm{H}_{2}(\sim 100 / 10 \mathrm{sccm})$ carrier gases), confirming the formation of near triangular $\mathrm{TaS}_{2}$ flakes. $\mathbf{f}$ Corresponding SEM image of as-grown $2 \mathrm{H}-\mathrm{TaS}_{2}$ flakes on Au foils. $\mathbf{g}$ AFM image and corresponding height profile of transferred $2 \mathrm{H}-\mathrm{TaS}_{2}$ flakes on $\mathrm{SiO}_{2} / \mathrm{Si}$ showing a nominal thickness of $\sim 3 \mathrm{~nm}$. $\mathbf{h}$ Large-area ultrathin $2 \mathrm{H}$-TaS $\mathrm{S}_{2}$ film evolved on Au foils by further prolonging the growth time to $20 \mathrm{~min}$ (with the other parameters keep identical to that of $\mathbf{c}, \mathbf{f}$ ). i AFM height image of a transferred film edge presenting the same thickness as the initially evolved flakes, as evidenced by the inset height profile analysis $(\sim 3 \mathrm{~nm})$. $\mathbf{j}$ Large-area OM image indicating the centimetre-size uniformity of the transferred $2 \mathrm{H}-\mathrm{TaS}_{2}$ film on $\mathrm{SiO}_{2} / \mathrm{Si}$ (synthesized at $750{ }^{\circ} \mathrm{C}$ for 20 min under $\mathrm{Ar} / \mathrm{H}_{2}(\sim 100 / 10$ sccm) carrier gases). Inset is the photograph of $2 \mathrm{H}-\mathrm{TaS} 2$ film on wafer-scale $\mathrm{SiO}_{2} / \mathrm{Si}$. Scales bars, $5 \mu \mathrm{m}$ in $\mathbf{c}-\mathbf{e}, 10 \mu \mathrm{m}$ in $\mathbf{f}, \mathbf{g}, 20 \mu \mathrm{m}$ in $\mathbf{h}, 50 \mu \mathrm{m}$ in $\mathbf{i}$ and $0.5 \mathrm{~mm}$ in $\mathbf{j}$ 

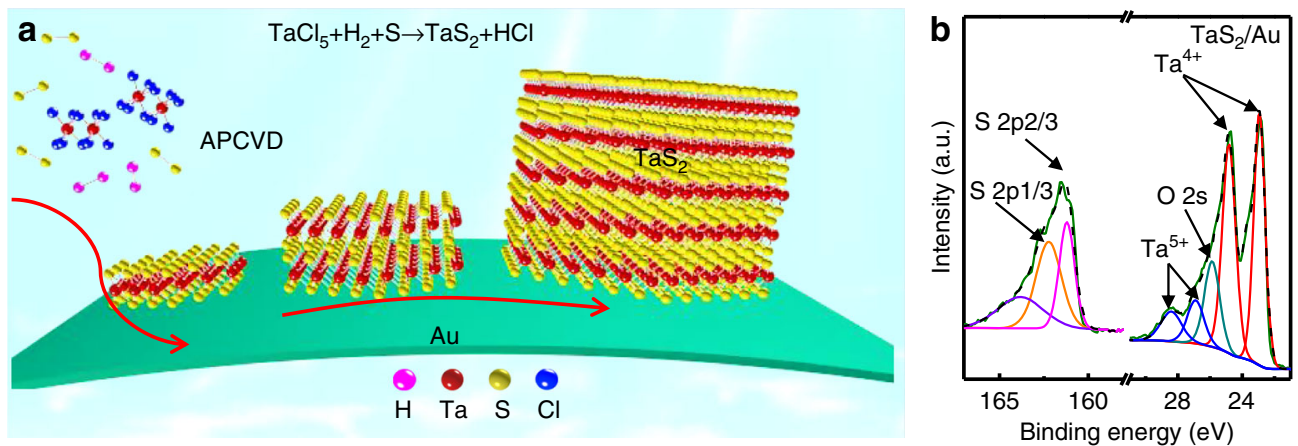

d

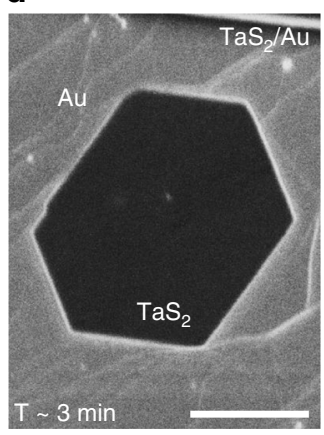

e

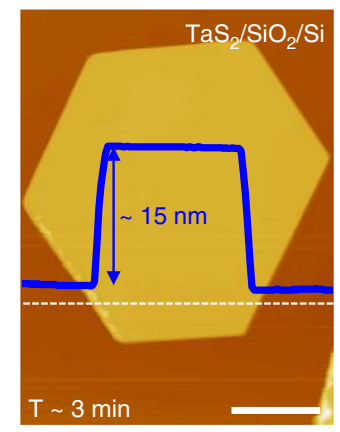

f

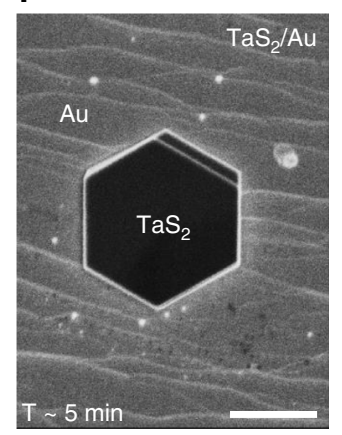

g

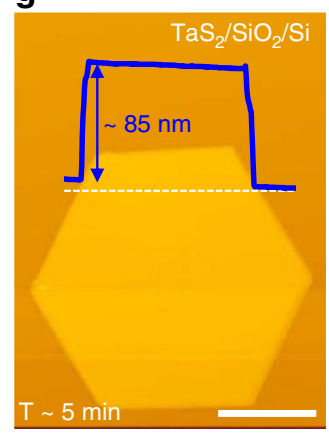

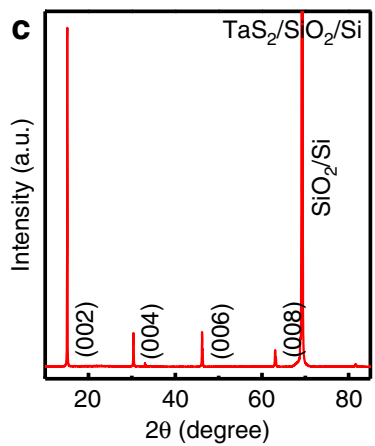

h

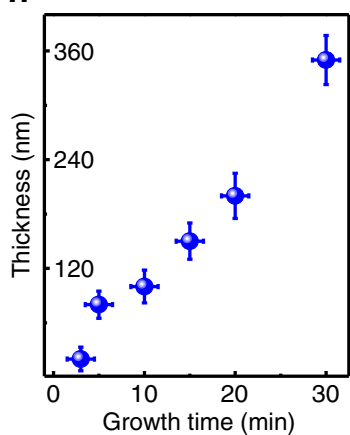

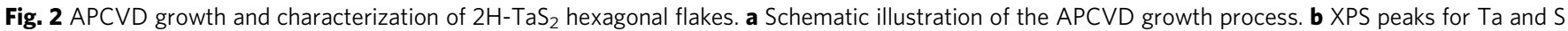
confirming the formation of $2 \mathrm{H}-\mathrm{TaS}_{2}$ on Au foils. c XRD pattern of transferred $2 \mathrm{H}-\mathrm{TaS}_{2}$ on $\mathrm{SiO}_{2} / \mathrm{Si}$ showing its $2 \mathrm{H}$ phase feature. $\mathbf{d}-\mathbf{g}$ SEM and corresponding AFM images showing the tunable thicknesses of hexagonal $2 \mathrm{H}-\mathrm{TaS}_{2}$ domains by varying growth time from 3 to $\sim 5$ min (synthesized at 750 ${ }^{\circ} \mathrm{C}$ under $\mathrm{Ar} / \mathrm{H}_{2}(\sim 100 / 10 \mathrm{sccm})$ carrier gases), respectively. $\mathbf{h}$ Plot of the thickness of $2 \mathrm{H}-\mathrm{TaS} 2$ as a function of growth time. Error bars are defined as s.d. Scale bars, $4 \mu \mathrm{m}$ in $\mathbf{d}$, e and $10 \mu \mathrm{m}$ in $\mathbf{f}, \mathbf{g}$

comparable with that of $1 \mathrm{~T}-\mathrm{MoS}_{2}{ }^{33}, 34$, presumably due to the slight oxidation of atomic-scale pores. Moreover, the use of a common glassy carbon working electrode possibly restricted the electron transfer from electrode to catalytically active sites, due to a weak interface interaction.

To tackle the above-mentioned issues, here we design lowpressure CVD (LPCVD) and APCVD routes for the direct syntheses of centimetre-sized uniform, ultrathin $\mathrm{TaS}_{2}$ films and thickness-tunable $\mathrm{TaS}_{2}$ flakes on a common electrode material of $\mathrm{Au}$ foil, respectively. This provides us with an opportunity to explore either fundamental physical phenomena or related applications associated with the dimensionality effect. In particular, the nearly commensurate CDW (NCCDW)/commensurate CDW (CCDW) phase transition is unambiguously demonstrated, suggesting that the crystalline quality of CVD-derived $\mathrm{TaS}_{2}$ is comparable to the mechanically exfoliated material. More significantly, the as-grown metallic $\mathrm{TaS}_{2}$ on $\mathrm{Au}$ foils displays high electrocatalytic activity for the HER. The internal reaction mechanism is revealed by a combination of experimental results and theoretical calculations.

\section{Results}

LPCVD synthesis of centimetre-sized ultrathin $2 \mathrm{H}-\mathrm{TaS}_{2}$ film. $\mathrm{TaS}_{2}$ thin films were successfully synthesized by a LPCVD route with solid $\mathrm{TaCl}_{5}$ and $\mathrm{S}$ as precursors, as depicted in the schematic view in Fig. 1a. In contrast to previous work reporting the synthesis of $\mathrm{TaS}_{2}$ flakes on $\mathrm{SiO}_{2} / \mathrm{Si}^{32}$, we selected $\mathrm{Au}$ foil as a substrates due to its chemical inertness towards S precursors, its catalytic activity in TMDCs growth, and more significantly, its compatibility with large-area growth and direct application in $\mathrm{HER}^{23}, 27$. X-ray photoemission spectroscopy (XPS) measurements were firstly carried out to determine the chemical composition of the as-grown samples (Fig. 1b, and Supplementary
Fig. 1). The obtained $\mathrm{Ta} 4 \mathrm{f}_{7 / 2}(22.7 \mathrm{eV})$ and $4 \mathrm{f}_{5 / 2}(24.7 \mathrm{eV})$ peaks are attributed to $\mathrm{Ta}^{4+}$, while the $\mathrm{S} 2 \mathrm{p}_{3 / 2}(162.1 \mathrm{eV})$ and $2 \mathrm{p}_{1 / 2}$ $(163.2 \mathrm{eV})$ peaks are assigned to $\mathrm{S}^{2-}$, in agreement with the standard XPS data of $\mathrm{TaS}_{2}{ }^{32}$. The Ta:S atomic ratio calculated from the XPS data is 1:2.08, approximating to the 1:2 stoichiometric ratio for bulk $\mathrm{TaS}_{2}$. Notably, additional peaks at 28.4 and $26.9 \mathrm{eV}$ are attributed to $\mathrm{Ta}^{5+}$, in consideration of the oxidation susceptibility of metallic $\mathrm{TaS}_{2}$.

Furthermore, synchrotron radiation-based low-energy electron microscopy (LEEM) and micro-beam XPS ( $\mu$-XPS) measurements were also performed directly on as-grown samples (Fig. 1c-e). Figure 1d, e reveal the spatial mapping of Ta $\left(4 \mathrm{f}_{7 / 2}\right)$ and $S\left(2 p_{3 / 2}\right)$, respectively, from which the shape/location of $\mathrm{TaS}_{2}$ flakes can be definitively distinguished. The uniform contrasts within the triangular domains indicate the relatively high crystal quality of the CVD-derived samples. The X-ray diffraction (XRD) pattern of $\mathrm{TaS}_{2}$ confirms its $2 \mathrm{H}$ phase structure (Supplementary Fig. 2), which is different from the previous report (1T-TaS synthesized on $\mathrm{SiO}_{2} / \mathrm{Si}$ with an APCVD route ${ }^{32}$ ). This difference can be explained by the relatively low growth temperature, the slow cooling process, and the different substrate used in this work. Notably, the $2 \mathrm{H}-\mathrm{TaS}_{2}$ should deliver a higher electrocatalytic activity than that of $1 \mathrm{~T}-\mathrm{TaS}_{2}$, as predicted by theoretical calculation $^{37}$

Scanning electron microscopy (SEM) examinations were then performed to show the morphology and the domain size evolution of $2 \mathrm{H}-\mathrm{TaS}_{2}$ on $\mathrm{Au}$ foils under different growth times of $\sim 5, \sim 10$, and $\sim 20 \mathrm{~min}$ (Fig. 1f, and Supplementary Fig. 3). The domain sizes were found to be variable from $\sim 0.5$ to $\sim 20 \mu \mathrm{m}$. In particular, at the growth time of $\sim 10 \mathrm{~min}$, the edge length of the $2 \mathrm{H}-\mathrm{TaS}_{2}$ triangle was as large as $\sim 20 \mu \mathrm{m}$, as shown in Fig. 1f. An apparent height of $\sim 3 \mathrm{~nm}$ was determined from the atomic force microscopy (AFM) section-view analysis across the domain edge 
a

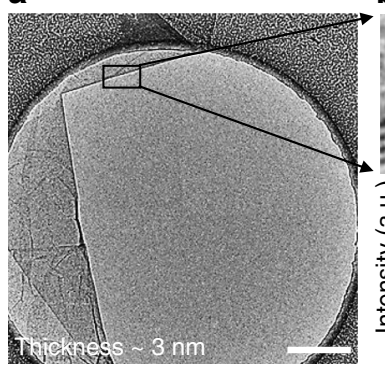

e

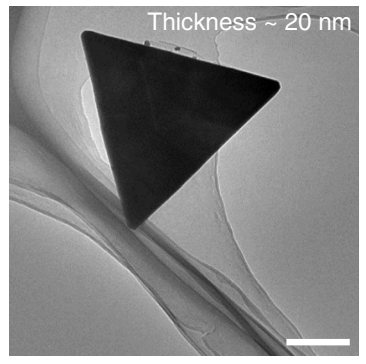

b

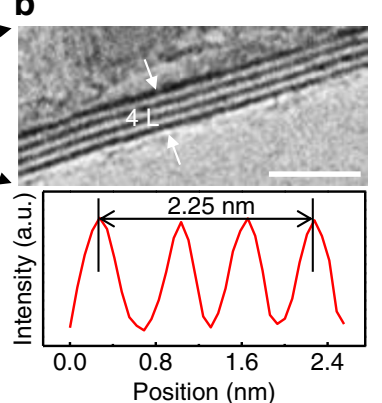

f

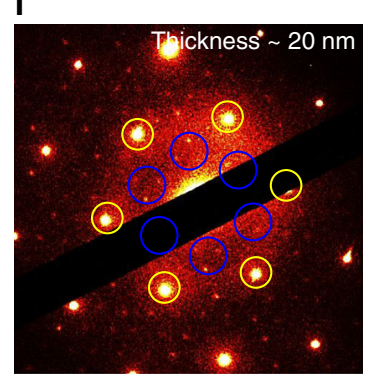

c

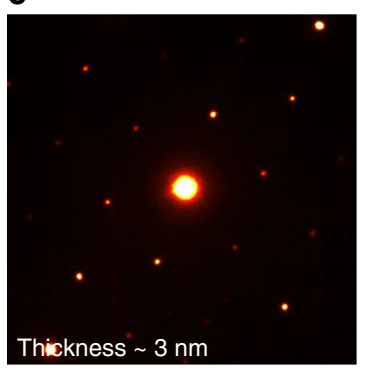

g

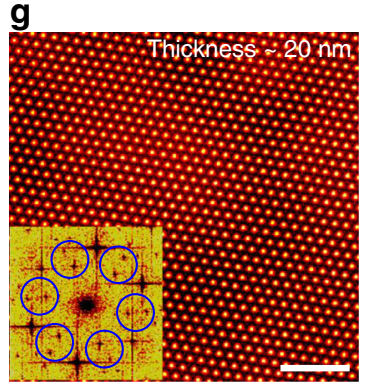

d

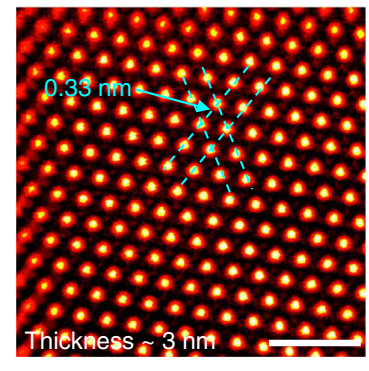

h

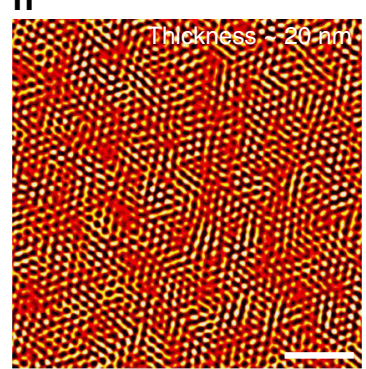

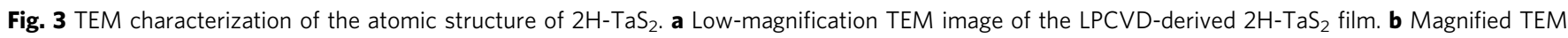

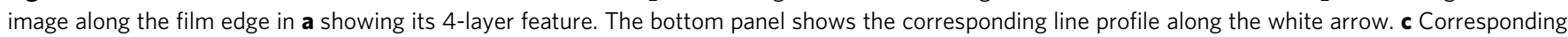
SAED pattern captured from a within a $500 \times 500 \mathrm{~nm}^{2}$ area. d Atomic-resolution TEM image of the transferred sample. e Low-magnification TEM image of a triangle domain with the thickness of $\sim 20 \mathrm{~nm}$. f Corresponding SAED pattern captured from e within a $2 \times 2 \mu \mathrm{m}^{2}$ area. $\mathbf{g}$ Atomic-resolution STEM-HAADF image showing the perfect atomic lattice. Inset is the corresponding FFT pattern. $\mathbf{h}$ Selective IFFT filtered image of NCCDW peaks in $\mathbf{g}$ ) showing disordered periodic lattice distortion. Scale bars, $1 \mu \mathrm{m}$ in $\mathbf{a}, \mathbf{e}, 3 \mathrm{~nm}$ in $\mathbf{b}, \mathbf{g}$, $\mathbf{h}$ and $1 \mathrm{~nm}$ in $\mathbf{d}$

of the transferred $2 \mathrm{H}-\mathrm{TaS}_{2}$ on $\mathrm{SiO}_{2} / \mathrm{Si}$ (Fig. 1g). However, at a growth time of $\sim 20 \mathrm{~min}$, a full coverage $2 \mathrm{H}-\mathrm{TaS}_{2}$ film was obtained according to the uniform SEM contrast in Fig. 1h. The AFM image of the transferred $2 \mathrm{H}-\mathrm{TaS}_{2}$ in Fig. 1i reveals a layer thickness of $\sim 3 \mathrm{~nm}$, the same as that achieved with reduced growth time (Fig. 1g). The excellent thickness uniformity at the centimetre-size was further confirmed by a highly homogeneous optical microscopy (OM) image of the transferred $2 \mathrm{H}-\mathrm{TaS}_{2}$ on $\mathrm{SiO}_{2} / \mathrm{Si}$ (Fig. 1j, and Supplementary Fig. 3). Note that, for other intermediate growth times, the thickness of the derived $2 \mathrm{H}-\mathrm{TaS}_{2}$ flakes maintained a similar value of $\sim 3 \mathrm{~nm}$. In this regard, we can infer that the current $2 \mathrm{H}-\mathrm{TaS}_{2}$ growth exhibits a 'magic' starting thickness of $\sim 3 \mathrm{~nm}$, i.e., individual islands evolved on the surface and then expanded with the increase of growth time, and finally merged together towards the formation of a complete layer. To the best of our knowledge, this is the first report about the synthesis of centimetre-sized uniform MTMDCs films.

It should be noted that the unique $2 \mathrm{D}$ growth feature is different from the self-limited surface growth of monolayer $\mathrm{MoS}_{2}$ or $\mathrm{WS}_{2}$ on $\mathrm{Au}$ foils 23,27 . We ascribe this 'magic' growth behaviour to the dimerization of Ta along the $c$-axis direction, as similarly demonstrated in an analogue system of $\operatorname{IrTe}_{2} 39,40$. A first-order structural transition from $1 \times 1 \times 1$ to $5 \times 1 \times 5$ was proposed for bulk $\mathrm{IrTe}_{2}$, and this reconstructed structure possessed a five-times periodicity in both $a$ and $c$ directions of the crystal lattice. The existence of long-range ordering along the $c$ direction (normal to the $2 \mathrm{D}$ plane) expressed an enhanced interplanar coupling with respect to traditional van der Waals coupled systems (i.e., $\mathrm{MoS}_{2}$ and $\left.\mathrm{WS}_{2}\right)^{39,40}$.

In order to confirm the aforementioned hypothesis, the growth time of $2 \mathrm{H}-\mathrm{TaS}_{2}$ was further prolonged to $\sim 30 \mathrm{~min}$. Some triangular $2 \mathrm{H}-\mathrm{TaS}_{2}$ flakes with the specific thickness of $\sim 3 \mathrm{~nm}$ were then observed on the complete 3 -nm-thick $2 \mathrm{H}-\mathrm{TaS}_{2}$ film (Supplementary Fig. 4). Such intriguing result strongly suggests the existence of a critical thickness for LPCVD synthesized $2 \mathrm{H}$ -
$\mathrm{TaS}_{2}$ on $\mathrm{Au}$ foils. Notably, this growth behaviour has also been reported in the "electronic growth" of metallic overlayers on semiconductor substrates, wherein quantized electronic states (QWSs) were generated in the thin layers and determined the stability of the $2 \mathrm{D}$ thin films ${ }^{41}$. Briefly, centimetre-sized uniform, several-layer-thick $2 \mathrm{H}-\mathrm{TaS}_{2}$ films were successfully obtained, which should offer attractive playgrounds for exploring some fundamental physical issues, e.g. the interplay between CDW and superconductivity that has been disclosed in mechanically exfoliated layers ${ }^{20}$

APCVD growth of $2 \mathrm{H}-\mathrm{TaS}_{2}$ flakes with tunable thickness. Recent electrical transport measurements (temperature-dependence resistance) have revealed that the thickness of exfoliated $\mathrm{TaS}_{2}$ flakes has a prominent influence on CCDW/NCCDW and NCCDW/incommensurate CDW phase transitions ${ }^{11}$. When the thickness was reduced to $\sim 3 \mathrm{~nm}$, such transitions suddenly vanished. In this regard, it is logical to synthesize high-quality, thickness-variable $\mathrm{TaS}_{2}$ so as to explore this thickness-dependent phenomenon. APCVD has proven to be an effective method to grow semiconducting TMDCs with tailored thicknesses, due to the excess precursor feeding rate during the synthesis process $^{42,43}$. Motivated by this, we selected the APCVD approach to synthesize $\mathrm{TaS}_{2}$ directly on $\mathrm{Au}$ foils, and the process is shown schematically in Fig. 2a. As a result, hexagonal $\mathrm{TaS}_{2}$ flakes were successfully achieved as presented in Supplementary Fig. 5. The morphology variations from triangular to hexagonal shapes between APCVD- and LPCVD-derived $\mathrm{TaS}_{2}$ are possibly attributed to the local changes of the Ta:S ratio of precursors, as previously demonstrated in $\mathrm{MoS}_{2}$ growth ${ }^{43}$. If the Mo:S ratio was larger than 1:2, triangular $\mathrm{MoS}_{2}$ domains were usually generated, however, when the Mo:S ratio was lower than 1:2, hexagonal $\mathrm{MoS}_{2}$ flakes were usually evolved. Notably, the stoichiometric ratio of $\mathrm{TaS}_{2}$ and its $2 \mathrm{H}$ phase structure were then confirmed by XPS and XRD measurements (Fig. 2b, c), the same as that of the 

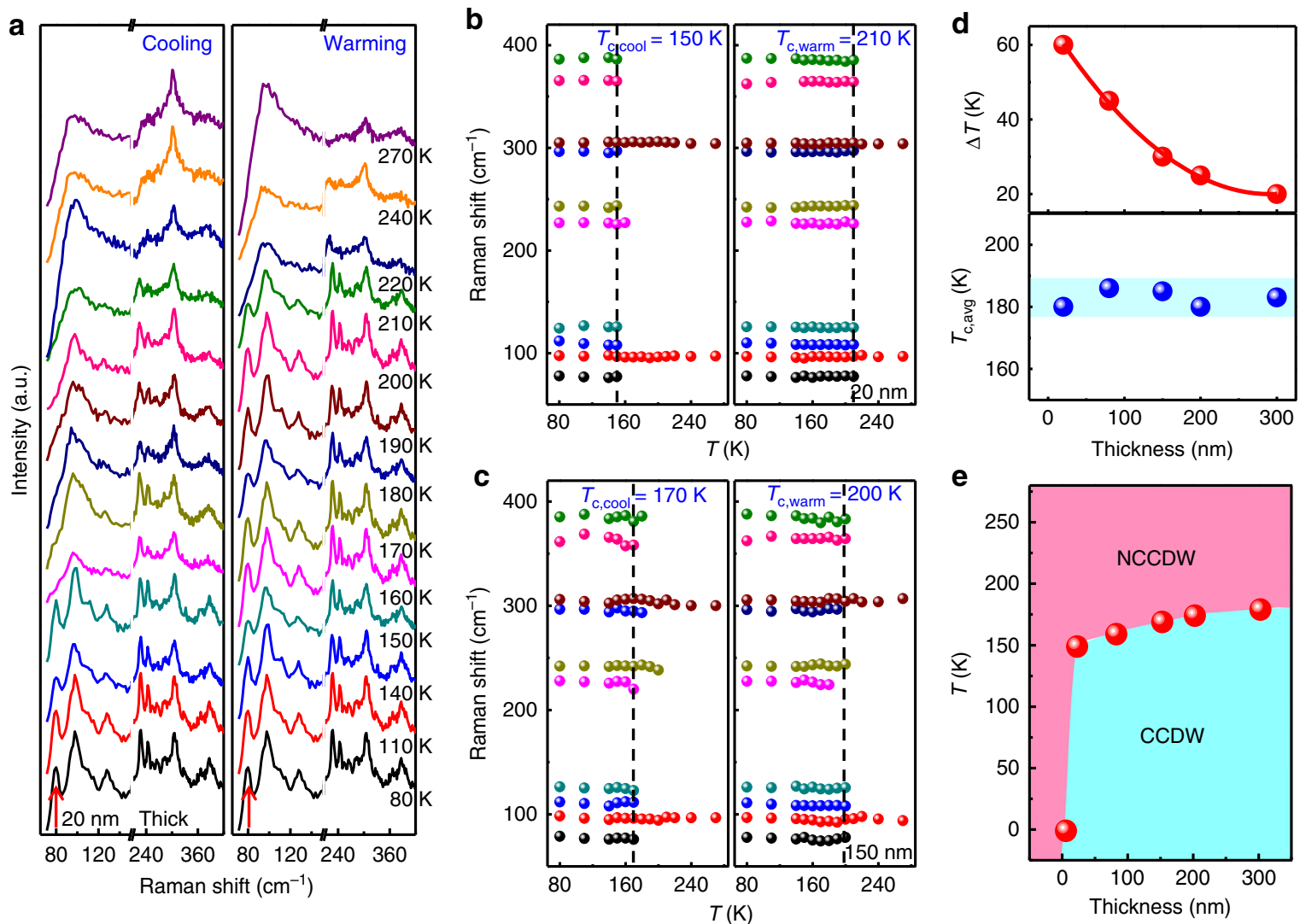

Fig. 4 Temperature-dependent Raman characterization of $2 \mathrm{H}-\mathrm{TaS}_{2}$ flakes with different thicknesses. a Temperature-dependent Raman spectra of $\sim 20 \mathrm{~nm}$-thick $2 \mathrm{H}-\mathrm{TaS}_{2}$ captured from both cooling and warming processes. b, c Raman frequency plots of discernible peaks for $\sim 20 \mathrm{~nm}$-thick and $\sim 150 \mathrm{~nm}$-thick $2 \mathrm{H}-\mathrm{TaS}_{2}$ flakes with decreasing/increasing temperature, respectively. $\mathbf{d}$ Hysteresis (upper) and average transition temperature (lower) plotted as a function of sample thickness. e Thickness-temperature phase diagram of CVD-derived $2 \mathrm{H}-\mathrm{TaS}_{2}$ obtained from temperature-dependent Raman data. The red balls mark the boundary of NCCDW and CCDW phases. For simplicity, the phase boundary was recorded based on the cooling data

LPCVD synthesized films. The XPS measurement of transferred $2 \mathrm{H}-\mathrm{TaS}_{2}$ on $\mathrm{SiO}_{2} / \mathrm{Si}$ was also performed to exclude the possible Au penetration into the $2 \mathrm{H}-\mathrm{TaS}_{2}$ layers during the $\mathrm{CVD}$ growth process (Supplementary Fig. 6).

Intriguingly, we found that upon increasing the growth time from $\sim 3$ to $\sim 30 \mathrm{~min}$, the edge length of the hexagonal $2 \mathrm{H}-\mathrm{TaS}_{2}$ flake can be tailored from $\sim 5$ to $\sim 20 \mu \mathrm{m}$ (Fig. $2 \mathrm{~d}, \mathrm{f}$ ) and the thickness from $\sim 15$ to $\sim 350 \mathrm{~nm}$ (Fig. 2e, g). This phenomenon highlights that the APCVD-synthesized $2 \mathrm{H}-\mathrm{TaS}_{2}$ on $\mathrm{Au}$ foils follows the Volmer-Weber (VW) growth mode, in contrast to the LPCVD growth obeying a Frank-van der Merwe (FM) mode. To provide further insight, the evolution of the flake thickness is plotted as a function of growth time (Fig. 2h), which clearly addresses the tunability of the thickness of $2 \mathrm{H}-\mathrm{TaS}_{2}$ by precisely varying the growth time. Altogether, high-quality, thicknesstunable, large-domain $2 \mathrm{H}-\mathrm{TaS}_{2}$ flakes can be synthesized on $\mathrm{Au}$ foils by an APCVD route.

TEM characterization of $2 \mathrm{H}-\mathrm{TaS}_{2}$. In order to obtain a thorough understanding of the crystal structure of the CVD-derived $2 \mathrm{H}-\mathrm{TaS}_{2}$, high-resolution transmission electron microscopy (HR-TEM) measurements were then performed on transferred samples. Figure 3a shows a low-magnification TEM image of the LPCVD-synthesized $2 \mathrm{H}-\mathrm{TaS}_{2}$, and the HR-TEM image captured from the film edge presents a layer thickness of 4 , as well as an interlayer spacing of $\sim 0.75 \mathrm{~nm}$ (Fig. 3b, and Supplementary Fig. 7). This data again confirms the existence of a 'magic' starting layer of 4 , which we believe was mediated by the dimerization of Ta along the $c$-axis direction. Moreover, the corresponding selected area electron diffraction (SAED) pattern in Fig. 3c reveals only one set of hexagonally arranged diffraction spots, strongly suggesting the single-crystalline nature of the $2 \mathrm{H}-\mathrm{TaS}_{2}$ domain. Atomic-resolution TEM image in Fig. 3d clearly displays a honeycomb structure with an interatomic distance of $\sim 0.33 \mathrm{~nm}$, as in good agreement with the documented lattice constant of $\mathrm{TaS}_{2}{ }^{20}$, further convincing the rather high crystalline quality of CVDderived $\mathrm{TaS}_{2}$.

Previous electrical transport measurements (temperaturedependent resistance) and TEM characterizations revealed that the CDW phase transitions of exfoliated $\mathrm{TaS}_{2}$ were strongly suppressed at a reduced thickness of $\sim 3 \mathrm{~nm}^{11}$, 13 . In order to observe the CDW phase transition in $2 \mathrm{H}-\mathrm{TaS}_{2}$, the APCVD samples were characterized by TEM and spherical-aberrationcorrected scanning transmission electron microscopy (STEM), a useful method to identify the CDW phases. The lowmagnification TEM image in Fig. 3e shows a representative $2 \mathrm{H}-$ $\mathrm{TaS}_{2}$ triangle of $\sim 20 \mathrm{~nm}$ thick. Notably, the corresponding SAED pattern acquired at room temperature is rather complex (Fig. 3f). The bright spots (highlighted by yellow circles) correspond to the Bragg scattering from the triangular lattice of $\mathrm{Ta}$ atoms with a lattice constant $a=0.33 \mathrm{~nm}$. This hexagonally arranged Bragg scattering pattern further confirms the single-crystalline feature of the $2 \mathrm{H}-\mathrm{TaS}_{2}$ triangle. However, the additional set of spots (indicated by blue circles surrounding the central beam) corresponds to the periodic lattice distortion (PLD) induced wave vectors, possibly due to the periodic atomic displacements of the NCCDW, as similarly reported for the exfoliated 1T$\mathrm{TaS}_{2}{ }^{13}$. 

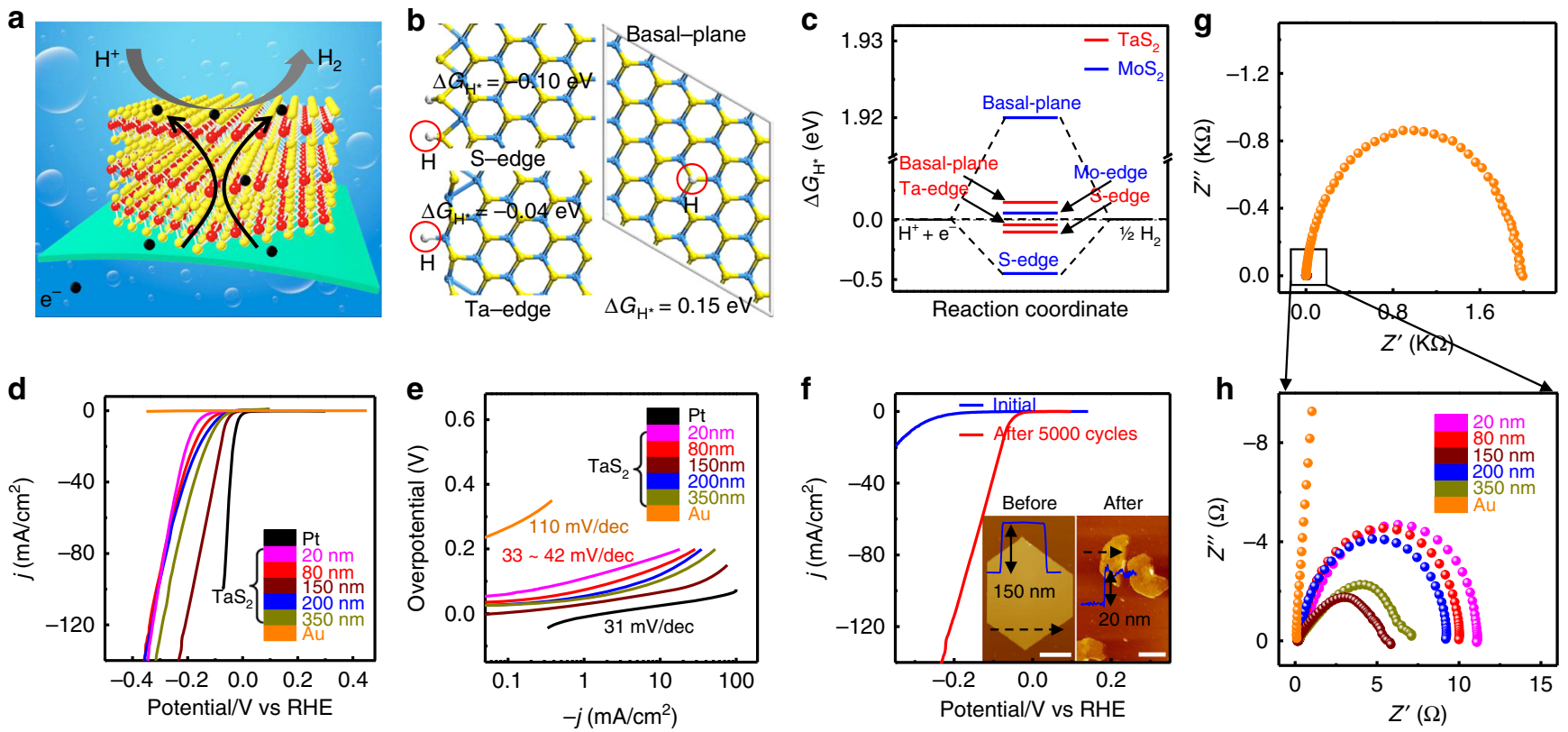

Fig. 5 Electrocatalytic application of CVD synthesized $2 \mathrm{H}-\mathrm{TaS}_{2}$ in HER. a Schematic illustration of the HER process of $2 \mathrm{H}-\mathrm{TaS} / \mathrm{Au}$ foils. b Hydrogen adsorption energies at S-edge, Ta-edge, and basal-plane of $2 \mathrm{H}-\mathrm{TaS}_{2}$, respectively. Yellow, cyan, and grey balls represent $\mathrm{S}, \mathrm{Ta}$, and adsorbed $\mathrm{H}$ atoms, respectively. $\mathbf{c} \Delta G_{\mathrm{H}^{*}}$ diagram of different $\mathrm{H}$ adsorption states. d Polarization curves (iR-corrected) of as-grown $2 \mathrm{H}$-TaS $\mathrm{S}_{2}$ with different thicknesses, Au foil, and commercial Pt. e Corresponding Tafel plots of the different samples in d. f Polarization curves (iR-corrected) of $2 \mathrm{H}-\mathrm{TaS}_{2}(\sim 150 \mathrm{~nm}$ thick) before and after 5000 cycles. Inset is the corresponding AFM images. $\mathbf{g}$, $\mathbf{h}$ Electrochemical impedance spectra of $2 \mathrm{H}-\mathrm{TaS}_{2}$ flakes with different thicknesses, as well as the Au foil substrate. Scale bars, $4 \mu \mathrm{m}$ in (f, left) and $2 \mu \mathrm{m}$ in (f, right)

In order to visualize the atomic-scale morphology and the NCCDW structure of $2 \mathrm{H}-\mathrm{TaS}_{2}$, atomic-resolution Z-contrast STEM-HAADF analysis was then carried out on the transferred sample. Figure $3 \mathrm{~g}$ reveals a representative STEM-HAADF image obtained from the $2 \mathrm{H}-\mathrm{TaS}_{2}$ triangle in Fig. 3e. The Ta (bright spots) and $\mathrm{S}$ atoms can be clearly identified by their different contrasts. However, the $S$ atoms are nearly invisible due to the large difference of the atomic number between Ta and S. Notably, the atomic arrangement obeys the $2 \mathrm{H}$-phase atomic model with $\mathrm{Ta}$ atoms octahedrally coordinated by $\mathrm{S}$ atoms, as inferred by the intensity line profile in Supplementary Fig. 7. A further selective inverse fast Fourier transform (IFFT) filtered image of the diffraction spots (highlighted by blue circles) in Fig. 3g shows disordered PLD, suggesting the appearance of NCCDW phase state in $\mathrm{TaS}_{2}$ (Fig. 3h). Briefly, the CVD-synthesized $2 \mathrm{H}-\mathrm{TaS}_{2}$ possesses comparable crystalline quality with that of mechanically exfoliated samples, which should allow more intensive investigations of physical phenomena such as CDW, superconductivity, and so on.

Thickness-dependent CDW phase transitions of $2 \mathrm{H}-\mathrm{TaS}_{2}$. The effect of dimensionality and interlayer coupling of $\mathrm{TaS}_{2}$ on the $\mathrm{CDW}$ phase transition has aroused interest ${ }^{11-}{ }^{13}$. However, the existing electrical transport measurements (temperature-dependent resistance) and TEM analyses are usually time-consuming and complex, and inefficient in distinguishing the CDW phases arising from either bulk or sample surface. Raman spectroscopy has been established as an exquisitely sensitive and convenient technique to investigate both bulk and surface vibration modes of $\mathrm{TMDCs}^{21,22}$. Very recently, temperature-dependent Raman spectroscopy has been utilized to determine the transition temperature of the CDW phase based on exfoliated $1 \mathrm{~T}-\mathrm{TaS}_{2}$ flakes $^{44}$.

In our work, representative Raman spectra have been captured on a $\sim 20 \mathrm{~nm}$-thick $2 \mathrm{H}-\mathrm{TaS}_{2}$ flake upon cooling/warming processes, as shown in Fig. 4a. For the cooling process, a broad
Raman peak was clearly observed below $100 \mathrm{~cm}^{-1}$ at $>150 \mathrm{~K}$. In contrast, some fine peaks (indicated by a red arrow in Fig. 4a) are visible below $100 \mathrm{~cm}^{-1}$ at $<150 \mathrm{~K}$, suggesting that a NCCDW/CCDW phase transition takes place at $\sim 150$ K. Notably, a similar tendency to change is also recorded during the warming process but with a higher transition temperature of $\sim 210 \mathrm{~K}$. Therefore, the critical temperature of NCCDW/CCDW phase transition is identified as $\sim 150$ and $\sim 210 \mathrm{~K}$ for the cooling and heating processes, respectively. In order to precisely determine the transition temperature of NCCDW/CCDW phase, Raman frequencies of discernible peaks as a function of temperature are plotted in Fig. 4b, upon cooling/warming processes. Obviously, the number of vibration modes and their frequencies are dramatically changed at the transition temperature $T_{\mathrm{c}}$ (marked by dashed lines), and this temperature is different between the cooling $\left(T_{c, \text { cool }}=150 \mathrm{~K}\right)$ and the warming $\left(T_{c, \text { warm }}=210 \mathrm{~K}\right)$ processes. The hysteresis temperature of $\Delta T=T_{c, w a r m}-T_{c, \text { cool }}$ $=60 \mathrm{~K}$ and the average transition temperature of $T_{\mathrm{c}, \text { avg }}=\left(T_{\mathrm{c}, \text { warm }}\right.$ $\left.+T_{c, \text { cool }}\right) / 2=180 \mathrm{~K}$ are then calculated accurately.

More temperature-dependent Raman spectra of $2 \mathrm{H}-\mathrm{TaS}_{2}$ with different thicknesses upon cooling/warming processes are presented in Supplementary Figs. 8 and 9. Interestingly, for the $\sim 3$ $\mathrm{nm}$-thick $2 \mathrm{H}-\mathrm{TaS}_{2}$, we find a negligible variation for the discernible peaks under different temperature, suggesting that the $\mathrm{CDW}$ phase transitions of $2 \mathrm{H}-\mathrm{TaS}_{2}$ are strongly suppressed at this ultrathin thickness region (Supplementary Fig. 8). Temperature-dependent Raman frequencies of discernible peaks for $2 \mathrm{H}-\mathrm{TaS}_{2}$ of $\sim 150 \mathrm{~nm}$ thick are also plotted in Fig. 4c, regarding cooling/warming processes. Contrastingly, Fig. 4d displays the hysteresis and average transition temperature plotted as a function of flake thickness. Apparently, $\Delta T$ increases with the reduction of sample thickness, while the $T_{c, \text { avg }}$ values are not changed substantially. As the film thickness decreases, the NCCDW/CCDW phase transition temperature decreases and vanishes at the critical thickness of $\sim 3 \mathrm{~nm}$, as presented in Fig. $4 \mathrm{e}$. Shortly, such abovementioned results are in good agreement with 
Table 1 Comparison of the HER performances of $2 \mathrm{H}-\mathrm{TaS}_{2}, 2 \mathrm{H}-\mathrm{MoS}_{2}$, and 1T-WS

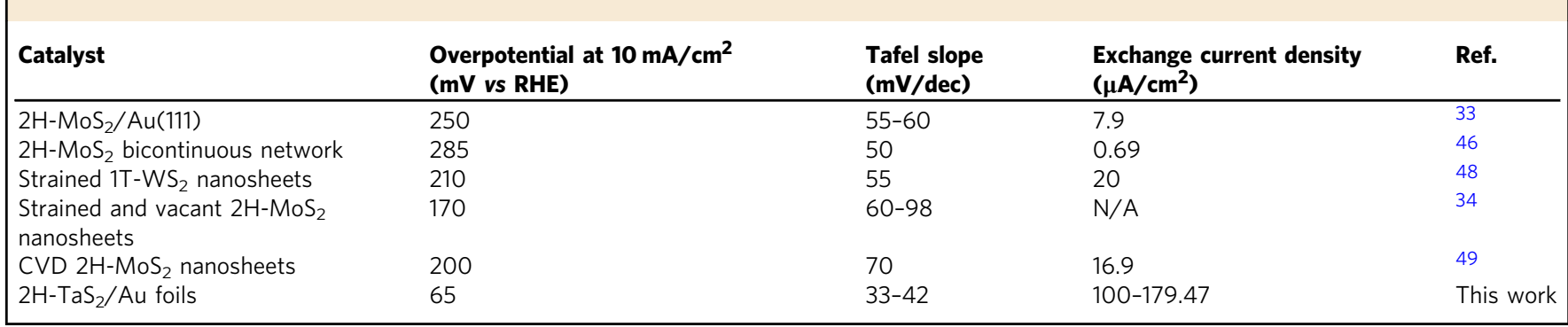

those of electrical transport measurements (temperature-dependent resistance) performed on exfoliated $\mathrm{TaS}_{2}{ }^{45}$, thus confirming the reliability of CVD-synthesized samples for detecting CDW phase transitions.

Electrocatalytic performance of $2 \mathrm{H}-\mathrm{TaS}_{2}$. A recent theoretical calculation predicted excellent electrocatalytic properties for metallic $2 \mathrm{H}-\mathrm{TaS}_{2}$ in HER, featuring high stability and active sites concentrated at the edges and in the basal-planes ${ }^{33}$. However, the direct application of $2 \mathrm{H}-\mathrm{TaS}_{2}$ in HER still remains unaddressed. Herein, the as-grown $2 \mathrm{H}-\mathrm{TaS}_{2}$ flakes on Au foils are directly used as electrocatalysts in HER, as schematically illustrated in Fig. 5 a. In order to justify the catalytically active sites of $2 \mathrm{H}-\mathrm{TaS}_{2}$, density functional theory (DFT) calculations are firstly performed (Fig. 5b, c). The Gibbs free energy $\left(\Delta G_{\mathrm{H}^{*}}\right)$ is usually used to assess the catalytic performance, and a $\Delta G_{\mathrm{H}^{*}}$ value close to zero usually indicates superior HER activity due to the optimal balance between absorption and removal of hydrogen atoms on the active sites $^{46}$. In Fig. $5 c$, the values of $\Delta G_{\mathrm{H}^{*}}$ for Ta-edge, S-edge, and basal-plane are calculated to be $-0.04,-0.10$, and $0.15 \mathrm{eV}$, respectively, which are all comparable with that of the $\mathrm{MoS}_{2}$ edge but remarkably lower than that of the $\mathrm{MoS}_{2}$ basal plane ${ }^{46}$. These calculated results indicate that the active sites of $2 \mathrm{H}-\mathrm{TaS}_{2}$ are concentrated both at the edges and in the basal-planes, in sharp contrast with that of $\mathrm{MoS}_{2}$ that posseses inert surface catalytic properties ${ }^{37}$. It is worth mentioning that, Yakobson, B. I. et al. have demonstrated that the populated state of electrons near the lowest unoccupied state $\left(\xi_{\text {LUS }}\right)$ is the key parameter of the adsorption strength of hydrogen on $\mathrm{MX}_{2}$ surfaces. The basalplane of $2 \mathrm{H}-\mathrm{TaS}_{2}$ possesses a relative low $\xi_{\mathrm{LUS}}(<-5.8 \mathrm{eV})$, thus possessing relatively strong adsorption capability of hydrogen and thus enhanced catalytic activity ${ }^{47}$.

Figure $5 \mathrm{~d}$ displays the polarization curves of as-grown $2 \mathrm{H}-\mathrm{TaS}_{2}$ flakes with different thicknesses (all the tested samples show similar coverage of $\sim 70 \%)$. The curves from $\mathrm{Au}$ foil and commercial Pt are also collected for comparison. Notably, at a cathodic current density $(j)$ of $10 \mathrm{~mA} / \mathrm{cm}^{2}$, the overpotentials $(\eta)$ of $2 \mathrm{H}-\mathrm{TaS}_{2}$ samples are falling in $65-150 \mathrm{mV}$, much lower than that of $\mathrm{MoS}_{2}(170-250 \mathrm{mV})^{33-36}$, possibly addressing the excellent HER activity of $2 \mathrm{H}-\mathrm{TaS}_{2}$. Furthermore, the linear portions of the Tafel plots in Fig. 5e are fitted to the Tafel equation $(\eta=b \log j+a$, where $j$ is the current density and $b$ is the Tafel slope), yielding Tafel slopes of 31,33-42, and $110 \mathrm{mV} / \mathrm{dec}$ for $\mathrm{Pt}, 2 \mathrm{H}-\mathrm{TaS}_{2} / \mathrm{Au}$, and $\mathrm{Au}$ foil, respectively. It is noteworthy that the Tafel slope $(\sim 33 \mathrm{mV} / \mathrm{dec})$ for $2 \mathrm{H}-\mathrm{TaS}_{2}$ with the thickness of $\sim 150 \mathrm{~nm}$ is very close to that of $\mathrm{Pt}(\sim 31 \mathrm{mV} / \mathrm{dec})$ and exceeds all the reported $\mathrm{MX}_{2}$ candidates $36,46,48,49$. The specific Tafel slope value should address a Volmer-Tafel mechanism for the HER of $2 \mathrm{H}-\mathrm{TaS}_{2}$.

By applying an extrapolation method to the Tafel plots, the exchange current density $\left(j_{0}\right)$ is also obtained and displayed in Supplementary Fig. 10. A remarkable $j_{0}$ value of $\sim 179.47 \mu \mathrm{A} / \mathrm{cm}^{2}$ is achieved, which is superior to other $\mathrm{MX}_{2}$ materials reported elsewhere $36,46,48,49$. To address this, a comparison of the HER performances of CVD-derived $2 \mathrm{H}-\mathrm{TaS}_{2}$ and $\mathrm{MX}_{2}$-based catalysts is displayed in Table 1 . Particularly, after 5000 cycles, the $2 \mathrm{H}$ $\mathrm{TaS}_{2}$ flakes present much higher electrocatalytic activity than that of their initial states (Fig. 5f, and Supplementary Fig. 11). Notably, $\mathrm{TaS}_{2}$ is a metallic TMDCs material, it is unstable under ambient condition and the surface can be oxidized, which results in extra low electrocatalytic activity for $\mathrm{HER}^{50}$. Through a facile HER cycling process, the surface oxides can be peeled off by hydrogen bubbles and the intrinsic electrocatalytic activities of $2 \mathrm{H}-\mathrm{TaS}_{2}$ are presented subsequently. Meanwhile, a microscopic morphology analysis reveals that the enhanced HER performance is closely correlated to the morphological evolution of $2 \mathrm{H}-\mathrm{TaS}_{2}$. The comparison of SEM and AFM morphologies of $2 \mathrm{H}-\mathrm{TaS}_{2}$ before and after 5000 cycles (Fig. 5f, and Supplementary Fig. 12) indicates that the flakes become thinner, smaller, and more disperse, but with invariable chemical composition of $2 \mathrm{H}-\mathrm{TaS}_{2}$ (Supplementary Fig. 13). In order to rule out the effect of the possible Pt contamination on the HER performance of $2 \mathrm{H}-\mathrm{TaS}_{2}$, we have re-measured the HER performance of $2 \mathrm{H}-\mathrm{TaS}_{2} / \mathrm{Au}$ by using the carbon rod as the counter electrode, and performed the Nafion proton exchange membrane assisted electrochemical measurement, respectively. Similar catalytic results have been achieved among the different methods, indicative of the high electrocatalytic performance of $2 \mathrm{H}-\mathrm{TaS}_{2} / \mathrm{Au}$ foils (Supplementary Fig. 14, and Supplementary Table 1). In our opinion, the cycling induced morphology change has three beneficial effects on the catalytic activity: 1) Shortening the interlayer electron-transfer pathways at a thinned domain; 2) increasing the active surface area by improving the accessibility of protons to basal-plane active sites; 3 ) increasing the density of active sites at the flake edge of $2 \mathrm{H}-\mathrm{TaS}_{2}$, considering that the $\Delta G_{\mathrm{H}^{*}}$ values of both Taedge and S-edge are much closer to the thermo-neutral point than that of the basal-plane, and the edge sites are catalytically more active than that of the basal plane. Such conclusions are further confirmed by the electrochemical impedance spectra (ESI) (Supplementary Fig. 15), where a decrease in charge-transfer resistance is observed upon cycling. The extra low charge-transfer resistance $(5-11 \Omega)$ in $2 \mathrm{H}-\mathrm{TaS}_{2} / \mathrm{Au}$ indicates the fast charge transfer between $\mathrm{TaS}_{2}$ and Au (Fig. $5 \mathrm{~g}, \mathrm{~h}$ ). The effect of CVD synthesis temperature on the HER performance of $\mathrm{TaS}_{2}$ was also presented in Supplementary Fig. 16, where low synthesis temperature $\left(<800^{\circ} \mathrm{C}\right)$ has negligible effect on the HER performance of $\mathrm{TaS}_{2}$, and the high synthesis temperature $\left(>800^{\circ} \mathrm{C}\right)$ reduces the catalytic activity of $\mathrm{TaS}_{2}$. This can be explained from the generation of different phases.

\section{Discussion}

In summary, we have developed facile LPCVD and APCVD routes for synthesizing large-area uniform, thickness controllable $2 \mathrm{H}-\mathrm{TaS}_{2}$ films and domains directly on Au foils, respectively. The high-quality $2 \mathrm{D} 2 \mathrm{H}-\mathrm{TaS}_{2}$ samples have proven to be attractive platforms for investigating fundamental physical phenomena (e.g. 
CDW) associated with the dimensionality effect. More significantly, the metallic $2 \mathrm{H}-\mathrm{TaS}_{2}$ have been found to be an efficient electrocatalyst for the HER, even comparable to Pt, owing to its abundant active sites concentrated at edges and basal-planes, as well as the self-optimizing morphological change of $2 \mathrm{H}-\mathrm{TaS}_{2}$. We believe this work could be a significant advance towards the batch production and electrocatalytic applications of $2 \mathrm{D}$ metallic materials, and hope these results will motivate scientists to explore new efficient catalysts in the large materials family of MTMDCs for energy related applications.

\section{Methods}

Materials synthesis. The $\mathrm{TaCl}_{5}$ (Alfa Aesar, purity 99.5\%) and S (Alfa Aesar, purity $99.5 \%$ ) powders were used as precursors and the Au foil as the substrate (Alfa Aesar, purity 99.99\%). All the sample growth was finished in a three-zone furnace (Lindberg/Blue M HTF55347c) equipped with a 1 inch diameter quartz tube. The temperature of $\mathrm{Au}$ foil, $\mathrm{TaCl}_{5}$ and S powders were set at 750,300 and $280^{\circ} \mathrm{C}$, respectively. $\mathrm{Ar}(100 \mathrm{sccm})$ and $\mathrm{H}_{2}(10 \mathrm{sccm})$ were used as carrier gases. After a growth period, the furnace was opened, and the sample was cooled to room temperature in the flowing mixed gases of $\mathrm{H}_{2} / \mathrm{Ar}(10 / 100 \mathrm{sccm})$.

Materials characterization. The samples were characterized by OM (Olympus BX51), SEM (Hitachi S-4800, 2 kV), XPS (Kratos Analytical AXIS-Ultra with monochromatic $\mathrm{Al} \mathrm{K \alpha}$ X-ray), XRD (Shimadzu Thin Film, using $\mathrm{Cu}$ Ko radiation at room temperature in the $2 \theta$ range of $10 \sim 90^{\circ}$ ), Raman spectroscopy (Renishaw, Invia Reflex, excitation light of $\sim 514 \mathrm{~nm}$ ), TEM (JEOL JEM-2100F LaB6; acceleration voltage, $200 \mathrm{kV}$ ), and AFM (Dimension Icon, Bruker). The LEEM and $\mu$-XPS elemental mapping data were acquired at the X-ray photoemission electron microscopy end station of the 09U (Dreamline) beamline of the Shanghai Synchrotron Radiation Facility. High resolution STEM-HAADF images were obtained on an aberration corrected transmission electron microscope JEM-ARM200F equipped with cold field emission gun with acceleration voltage of $200 \mathrm{kV}$.

Electrochemical measurements. All the electrochemical measurements were performed in a three-electrode system on $\mathrm{CHI} 760 \mathrm{E}$ electrochemical workstation ( $\mathrm{CH}$ Instruments), using $2 \mathrm{H}-\mathrm{TaS}_{2} / \mathrm{Au}$ foil as the working electrode, a Pt foil or carbon rod as a counter electrode, and a saturated $\mathrm{Ag} / \mathrm{AgCl}$ as a reference electrode. All the potentials were calibrated by a reversible hydrogen electrode (RHE). Linear sweep voltammetry with a scan rate of $\sim 5 \mathrm{mV} \mathrm{s}^{-1}$, from +0.10 to $-0.70 \mathrm{~V}$ vs. RHE was conducted in $0.5 \mathrm{M} \mathrm{H}_{2} \mathrm{SO}_{4}$ (sparged with $\mathrm{N}_{2}$, purity $\sim 99.999 \%$ ). The Nyquist plots were obtained with frequencies ranging from $100 \mathrm{kHz}$ to $0.1 \mathrm{~Hz}$ at the overpotential of $10 \mathrm{mV}$. The impedance data were fitted to a simplified Randles circuit to extract the series and charge-transfer resistances.

DFT calculations. All theoretical calculations were performed within the framework of DFT using the Vienna ab initio simulation package (VASP) ${ }^{51}$ with projector-augmented wave scheme. Then the Gibbs free energy for hydrogen adsorption, $\Delta G_{\mathrm{H}^{*}}$, was estimated following the procedure described in a previous report $^{52}$

Data availability. The data reported by this article are available from the corresponding author upon reasonable request.

Received: 25 April 2017 Accepted: 16 August 2017

Published online: 16 October 2017

\section{References}

1. Li, L. J. et al. Controlling many-body states by the electric-field effect in a twodimensional material. Nature 529, 185-189 (2016).

2. Porer, M. et al. Non-thermal separation of electronic and structural orders in a persisting charge density wave. Nat. Mater. 13, 857-861 (2014).

3. Joe, Y. I. et al. Emergence of charge density wave domain walls above the superconducting dome in 1T-TiSe 2 . Nat. Phys. 10, 421-425 (2014).

4. Peng, J. F. et al. Molecular beam epitaxy growth and scanning tunneling microscopy study of TiSe 2 ultrathin films. Phys. Rev. B 91, 121113 (2015).

5. Wang, J. Y. et al. Controlled synthesis of two-dimensional $1 \mathrm{~T}-\mathrm{TiSe}_{2}$ with charge density wave transition by chemical vapor transport. J. Am. Chem. Soc. 138, 16216-16219 (2016).

6. Langer, M. et al. Giant frictional dissipation peaks and charge-density-wave slips at the $\mathrm{NbSe}_{2}$ surface. Nat. Mater. 13, 173-177 (2014).

7. Ugeda, M. M. et al. Characterization of collective ground states in single-layer $\mathrm{NbSe}_{2}$. Nat. Phys. 12, 92-97 (2016).
8. Xi, X. X. et al. Strongly enhanced charge-density-wave order in monolayer $\mathrm{NbSe}_{2}$. Nat. Nanotechnol. 10, 765-769 (2015).

9. Xi, X. X. et al. Ising pairing in superconducting $\mathrm{NbSe}_{2}$ atomic layers. Nat. Phys 12, 139-143 (2016).

10. Sipos, B. et al. From Mott state to superconductivity in $1 \mathrm{~T}-\mathrm{TaS}_{2}$. Nat. Mater. 7 , 960-965 (2008)

11. Yu, Y. J. et al. Gate-tunable phase transitions in thin flakes of $1 \mathrm{~T}-\mathrm{TaS}_{2}$. Nat. Nanotechnol. 10, 270-276 (2015).

12. Liu, J. X. et al. A charge-density-wave oscillator based on an integrated tantalum disulfide-boron nitride-graphene device operating at room temperature. Nat. Nanotechnol. 11, 845-850 (2015).

13. Hovden, R. et al. Atomic lattice disorder in charge-density-wave phases of exfoliated dichalcogenides (1T-TaS 2 ). Proc. Natl. Acad. Sci. USA 113, 11420-11424 (2016).

14. van Wezel, J. et al. Effect of charge order on the plasmon dispersion in transition-metal dichalcogenides. Phys. Rev. Lett. 107, 176404 (2011).

15. Samnakay, R. et al. Zone-folded phonons and the commensurate-incommensurate charge-density-wave transition in $1 \mathrm{~T}-\mathrm{TaSe}_{2}$ thin films. Nano. Lett. 15, 2965-2973 (2015).

16. Sun, S. S. et al. Direct observation of an optically induced charge density wave transition in 1T-TaSe 2 . Phys. Rev. B 92, 224303 (2015).

17. Song, C. L. et al. Direct observation of nodes and twofold symmetry in FeSe superconductor. Science 17, 1410-1413 (2015).

18. Zhang, T. et al. Superconductivity in one-atomic-layer metal films grown on $\mathrm{Si}$ (111). Nat. Phys. 6, 104-108 (2010).

19. Li, W. et al. Phase separation and magnetic order in K-doped iron selenide superconductor. Nat. Phys. 8, 126-130 (2012).

20. Navarro-Moratalla, E. et al. Enhanced superconductivity in atomically thin $\mathrm{TaS}_{2}$. Nat. Commun. 7, 11043 (2016).

21. Lee, Y. et al. Synthesis of large-area $\mathrm{MoS}_{2}$ atomic layers with chemical vapor deposition. Adv. Mater. 24, 2320-2325 (2012).

22. Shi, Y. M. et al. van der Waals epitaxy of $\mathrm{MoS}_{2}$ layers using graphene as growth templates. Nano. Lett. 12, 2784-2791 (2012).

23. Shi, J. P. et al. Controllable growth and transfer of monolayer $\mathrm{MoS}_{2}$ on $\mathrm{Au}$ foils and its potential application in hydrogen evolution reaction. ACS Nano 8 , 10196-10204 (2014)

24. Chang, Y. H. et al. Monolayer $\mathrm{MoSe}_{2}$ grown by chemical vapor deposition for fast photodetection. ACS Nano 8, 8582-8590 (2014).

25. Lu, X. et al. Large-area synthesis of monolayer and few-layer $\mathrm{MoSe}_{2}$ films on $\mathrm{SiO}_{2}$ substrates. Nano. Lett. 14, 2419-2425 (2014).

26. Zhang, Y. et al. Controlled growth of high-quality monolayer $\mathrm{WS}_{2}$ layers on sapphire and imaging its grain boundary. ACS Nano 7, 8963-8971 (2013).

27. Gao, Y. et al. Large-area synthesis of high-quality and uniform monolayer $\mathrm{WS}_{2}$ on reusable Au foils. Nat. Commun. 6, 8569 (2015).

28. Keyshar, K. et al. Chemical vapor deposition of monolayer rhenium disulfide $\left(\mathrm{ReS}_{2}\right)$. Adv. Mater. 19, 4640-4648 (2015).

29. Zhang, Q. et al. Extremely weak van der waals coupling in vertical $\mathrm{ReS}_{2}$ nanowalls for high-current-density lithium-ion batteries. Adv. Mater. 28, 2616-2623 (2016).

30. Yuan, J. T. et al. Facile synthesis of single crystal vanadium disulfide nanosheets by chemical vapor deposition for efficient hydrogen evolution reaction. $A d v$. Mater. 27, 5605-5609 (2015).

31. Ji, Q. Q. et al. Metallic vanadium disulfide nanosheets as a platform material for multifunctional electrode applications. Nano Lett. 17, 4908-4916 (2017).

32. $\mathrm{Fu}, \mathrm{W}$. et al. Controlled synthesis of atomically thin $1 \mathrm{~T}-\mathrm{TaS}_{2}$ for tunable charge density wave phase transitions. Chem. Mater. 28, 7613-7618 (2016).

33. Jaramillo, T. F. et al. Identification of active edge sites for electrochemical $\mathrm{H}_{2}$ evolution from $\mathrm{MoS}_{2}$ nanocatalysts. Science 317, 100-102 (2007).

34. Li, H. et al. Activating and optimizing $\mathrm{MoS}_{2}$ basal planes for hydrogen evolution through the formation of strained sulphur vacancies. Nat. Mater. 15 48-53 (2016).

35. Yu, Y. F. et al. Layer-dependent electrocatalysis of $\mathrm{MoS}_{2}$ for hydrogen evolution. Nano. Lett. 14, 553-558 (2014).

36. Voiry, D. et al. Conducting $\mathrm{MoS}_{2}$ nanosheets as catalysts for hydrogen evolution reaction. Nano. Lett. 13, 6222-6227 (2014).

37. Tsai, C., Chan, K., Nørskov, J. K. \& Abild-Pedersen, F. Theoretical insights into the hydrogen evolution activity of layered transition metal dichalcogenides. Surf. Sci. 640, 133-140 (2015).

38. Li, H. et al. Atomic-sized pores enhanced electrocatalysis of $\mathrm{TaS}_{2}$ nanosheets for hydrogen evolution. Adv. Mater. 28, 8945-8949 (2016).

39. Pascut, G. L. et al. Dimerization-induced cross-Layer quasi-two-dimensionality in metallic $\mathrm{IrTe}_{2}$. Phys. Rev. Lett. 112, 086402 (2014).

40. Chen, C. et al. Surface phases of the transition-metal dichalcogenide $\operatorname{IrTe}_{2}$. Phys. Rev. B 95, 094118 (2017)

41. Zhang, Z. Y., Niu, Q. \& Shih, C.-K. "Electronic growth" of metallic overlayers on semiconductor substrates. Phys. Rev. Lett. 80, 5381 (1998).

42. Ling, X. et al. Role of the seeding promoter in $\mathrm{MoS}_{2}$ growth by chemical vapor deposition. Nano. Lett. 4, 464-472 (2014). 
43. Najmaei, S. et al. Vapour phase growth and grain boundary structure of molybdenum disulphide atomic layers. Nat. Mater. 12, 754-759 (2013).

44. He, R. et al. Distinct surface and bulk charge density waves in ultrathin 1T$\mathrm{TaS}_{2}$. Phys. Rev. B 94, 201108 (2016). (R).

45. Tsen, A. W. et al. Structure and control of charge density waves in twodimensional 1T-TaS . Proc. Natl. Acad. Sci. USA 112, 15054-15059 (2015).

46. Kibsgaard, J., Chen, Z. B., Reinecke, B. N. \& Jaramillo, T. F. Engineering the surface structure of $\mathrm{MoS}_{2}$ to preferentially expose active edge sites for electrocatalysis. Nat. Mater. 11, 963-969 (2012).

47. Liu, Y. Y. et al. Self-optimizing, highly surface-active layered metal dichalcogenide catalysts for hydrogen evolution. Nat. Energy 6, 17127 (2017).

48. Voiry, D. et al. Enhanced catalytic activity in strained chemically exfoliated $\mathrm{WS}_{2}$ nanosheets for hydrogen evolution. Nat. Mater. 12, 850-855 (2013).

49. Voiry, D. et al. The role of electronic coupling between substrate and 2D $\mathrm{MoS}_{2}$ nanosheets in electrocatalytic production of hydrogen. Nat. Mater. 15, 1003-1009 (2016).

50. Awaludin, Z., Safuan, M., Okajima, T. \& Ohsaka, T. Investigating the physical and electrochemical effects of cathodic polarization treatment on $\mathrm{TaO}_{\mathrm{x}}$. J. Mater. Chem. A 3, 16791-16800 (2015).

51. Hinnemann, B. et al. Biomimetic hydrogen evolution: $\mathrm{MoS}_{2}$ nanoparticles as catalyst for hydrogen evolution. J. Am. Chem. Soc. 127, 5308-5309 (2005).

52. Kresse, G. \& Furthmüller, J. Efficient iterative schemes for ab initio total-energy calculations using a plane-wave basis set. Phys. Rev. B 54, 11169 (1996).

\section{Acknowledgements}

We gratefully acknowledge the financial support from the National Natural Science Foundation of China (Nos. 51290272, 51472008, 51472080, 51432002, 51520105003,

$51522212,51421002,51672307$, and 21673054), the Ministry of Science and Technology of China (Nos. 2016YFA0200103, 2016YFA0200700, 2013CB932603, and

2014CB921002), the Open Research Fund Program of the State Key Laboratory of Low Dimensional Quantum Physics (No. KF201601), the Strategic Priority Research Program of Chinese Academy of Sciences (No. XDB07030200), and the Key Research Program of Frontier Sciences, Chinese Academy of Sciences (Nos. QYZDB-SSW-JSC035, and QYZDB-SSW-SYS031).

\section{Author contributions}

J.S. and X.W. contributed equally to this work. Y.Z. conceived and supervised the research project. J.S. developed and conducted the CVD growth of $\mathrm{TaS}_{2}$, with Y.H., Z.Z., X.Z., M.H., and Q.F.'s assistance. Y.G. and L.G. performed the STEM-HAADF characterization. J.S., Y.H., Z.Z., X.Z., M.H., and Q.F. carried out the OM, XPS, XRD, SEM, AFM, TEM, LEEM and $\mu$-XPS characterization. X.W., Y.H., and L.X. performed the electrochemical measurements. S.Z., Q.Z., and X.L. carried out the temperaturedependent Raman spectroscopy characterization. Y.L. performed the DFT calculations. All the authors discussed the results and commented on the manuscript.

\section{Additional information}

Supplementary Information accompanies this paper at doi:10.1038/s41467-017-01089-z.

Competing interests: The authors declare no competing financial interests.

Reprints and permission information is available online at http://npg.nature.com/ reprintsandpermissions/

Publisher's note: Springer Nature remains neutral with regard to jurisdictional claims in published maps and institutional affiliations.

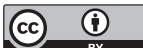

Open Access This article is licensed under a Creative Commons Attribution 4.0 International License, which permits use, sharing, adaptation, distribution and reproduction in any medium or format, as long as you give appropriate credit to the original author(s) and the source, provide a link to the Creative Commons license, and indicate if changes were made. The images or other third party material in this article are included in the article's Creative Commons license, unless indicated otherwise in a credit line to the material. If material is not included in the article's Creative Commons license and your intended use is not permitted by statutory regulation or exceeds the permitted use, you will need to obtain permission directly from the copyright holder. To view a copy of this license, visit http://creativecommons.org/ licenses/by/4.0/.

(C) The Author(s) 2017 